\title{
Design and Construction of a Novel Micro-Extrusion System for Bio- printing Applications
}

\author{
Levent Aydın * ${ }^{1}$, Assoc. Prof. Serdar Küçük ${ }^{1}$, Assoc. Prof. Halime Kenar ${ }^{1}$ \\ Accepted $3^{\text {rd }}$ September 2016
}

\begin{abstract}
Three-dimensional (3d) bio-printing is one of the major research fields of future, as yet at the beginning stage but producing promising solutions in medicine. As technology evolves, novel systems emerge for positioning even a single cell to the desired place to create functional tissues. The precision of these systems determine the functionality of outputs. In general, bio-printers use Ink-jet, Micro-extrusion and Laser Assisted printing methods to construct a solid tissue or a part of an organ. Ink-jet method, also known as dropon-demand bio-printing approach, is based on spraying cells by means of thermal or piezo electric pulses from numerous nozzles and is commonly used when forming tissues like skin and cartilage. Micro-extrusion method is used when complex biological structures like blood vessels or solid organs are bio-printed. Laser Assisted method is more preferred for biomaterial or implant production. Components of these systems have direct effects on the output since they determine where and how much biological material will be deposited in every layer. The aim of this study is to design and construct a novel micro-extrusion module for bio-printing applications. The designed module consists of three-dimensional (3d) printed body parts from Polylactic acid (PLA), Nema type stepper motors, ball screws (SFU1204), ball screw nuts (M12), flexible couplings (5 x 8 x $25 \mathrm{~mm}$ ), steel rods (M8), SCE UU series bearings (SCE 8 UU), UFL series bearings (UFL 08), LMEF series bearings (LMEF 8 UU), SK series rod holders (SK 08) and has an ability to control three commercially available syringes with blunt ended needles. For precise micro-extrusion, galvanized steel rods support ball screw driven linear motions. Ergonomically, syringes can be easily mount and locked and this system also has laser holders that can be used for targeted photo polymerization.
\end{abstract}

Keywords: Bio-printing, Micro-extrusion module, Three syringes, Photo polymerization.

\section{Introduction}

Three-dimensional (3d) bio-printing is based on biomaterial localization in limited conditions to construct functional tissues or a part of an organ generally using ink-jet, micro-extrusion and laser assisted bio-printing methods [1]. The applicability of these systems is similar to two-dimensional (2d) printers, however this technology uses biological materials instead of an ink [2]. The system of a bio-printer consists of electrical, mechanical and software components to carry out controlled biological material deposition [3]. Mechanical components such as main frame, bearings, rods and other parts have direct effect on the movement ability and determine the physical limits of the machine. Electrical parts are used to control these mechanics and can be mentioned as a mainboard, typical driven stepper or servo motors, mechanical or optic limit switches, thermistor or thermocouples, heaters and other human computer interfaces like LCD (Liquid Crystal Display) or keypad. The software can be separated as Firmware that is also embedded in MCU (Micro Controller Unit) and host software can be controlled via PC (Personal Computer). Firmware is a library that determines the whole system by means of loops, variables and values that have direct effects on efficiency. Although there are several differences between a milling, drilling or grinding machine and a $3 \mathrm{~d}$ bioprinter, the implementation of these systems and fundamental components have similarities. In fact, printing technology, print head and printed material are developed or chosen independently of one another when designing stage of these systems according

${ }^{1}$ Kocaeli University Kocaeli/Turkey

* Corresponding Author: Email: NONE

Note: This paper has been presented at the $3^{\text {rd }}$ International Conference on Advanced Technology \& Sciences (ICAT'16) held in Konya (Turkey), September 01-03, 2016.

This journal is @ Advanced Technology \& Science 2013 to the purposes [4]. Printouts are dependent on the precision of the whole microfabrication system and biomechanical characteristics of the bio-inks [5]. It should be noticed that additive manufacturing technology is accepted as a different way of producing things from the traditional methods, nevertheless they both have similar components [6]. The design of the system of interest is shaped by means of purposes that determine the limits of imagination.

\section{Basics of Bio-printing Technologies}

As technology evolves, novel systems emerge for positioning even a single cell to create functional tissues [7]. The precision of these systems determine the functionality of outputs. In general, bio-printers use Ink-jet, Micro-extrusion and Laser Assisted printing methods to construct a solid tissue or a part of an organ [8]. Ink-jet method uses generated droplets down to 20 microns in diameter via the magnitude of applied thermal or piezo-electrical forces [9]. As a result of spraying living cells from numerous nozzles, non-complex biological structures like skin and cartilage can easily be formed with ink-jet based printing. Figure 1 illustrates one of the numerous nozzles from a print head that uses ink-jet bio-printing approach. 

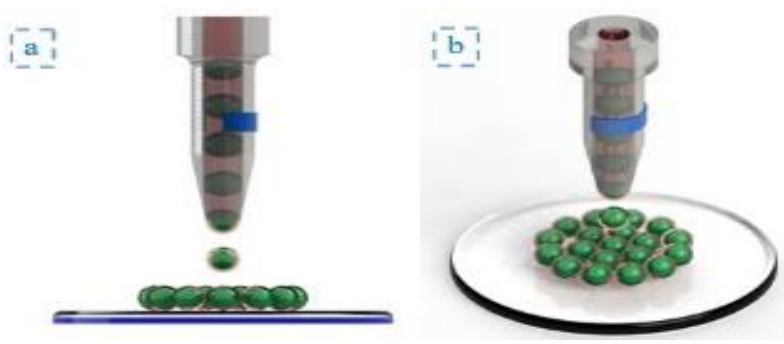

Figure 1. Ink-jet bio-printing approach, a) front view b) perspective view

Micro-extrusion method has an ability to form complex biological structures like blood vessels, heart valves and solid organs [10]. Extrusion of biomaterials can be performed by means of a piston, an extrusion screw or compressed air. All these print heads have similar continuous material deposition with different types of mechanisms. Syringes are used as hydrogel tanks and chosen according to the deposition technique. Blunt end needles are preferred as nozzles and diameters of needles depend on the cell sizes used in bio-printing process. Print heads and the biomaterials used should be chosen carefully in order to prevent shear stress of hydrogels that may occur during material deposition [11]. Shear stress can cause cell death that directly affects the functionality of output tissue constructs. Figure 2 illustrates the three deposition techniques used in microextrusion bio-printing approach.
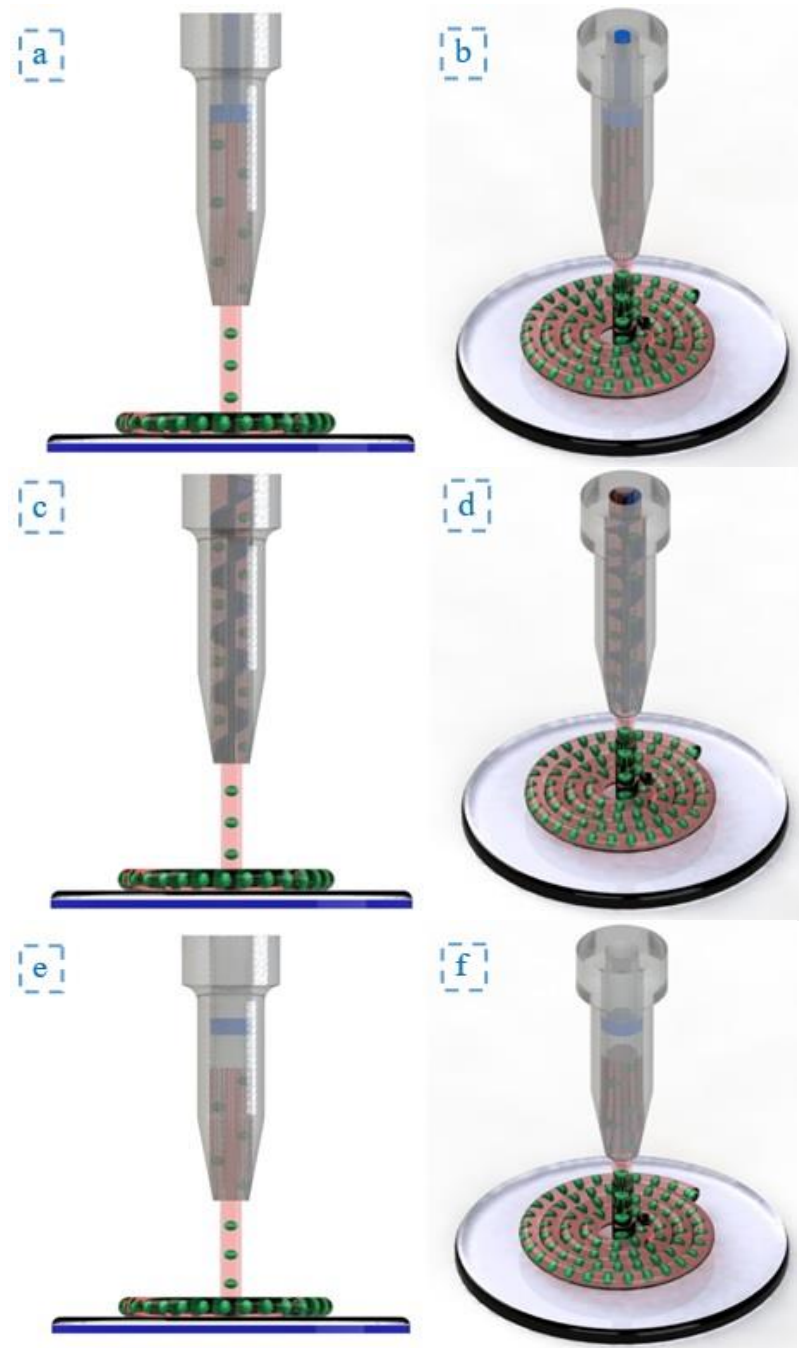

Figure 2. Micro-extrusion bio-printing approaches using a piston (front view (a) and perspective view (b)) an extrusion screw (front view (c) and perspective view (d)), and compressed air (front view (e) and perspective view (f)).
Laser assisted method is more preferred for implant production [12]. The wavelength of laser beam is critical for cell viability when biological materials are exposed and this has a direct effect on the output. In order to prevent cell death and improve output tissue's functionality, an energy absorbance layer is used. This method is based on selective biomaterial detachment from donor side to the substrate via laser pulses, and is the most expensive technique when compared with all techniques. Thus, laser assisted method is used for more precise applications. Figure 3 illustrates the laser assisted bio-printing approach.
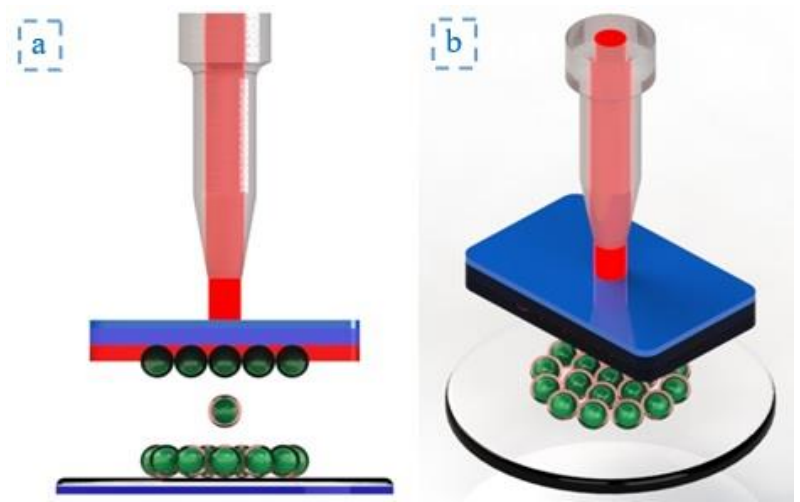

Figure 3. Laser assisted bio-printing approach, a) front view b) perspective view

\section{Material and Method}

Prototype manufacturing has stages extending from blue-prints to the end usage. Initially, target system is drawn as $2 \mathrm{~d}$ (twodimensional) sketches. The main frame or other parts are shaped according to the desired functionality. The model of design is taken to its final form by means of a $3 \mathrm{~d}$ Computer Aided Design (CAD) software. This helps to see the whole assembly and bill of materials (BOM).

Figure 4 illustrates the assembled parts of prototype on CAD software [13].

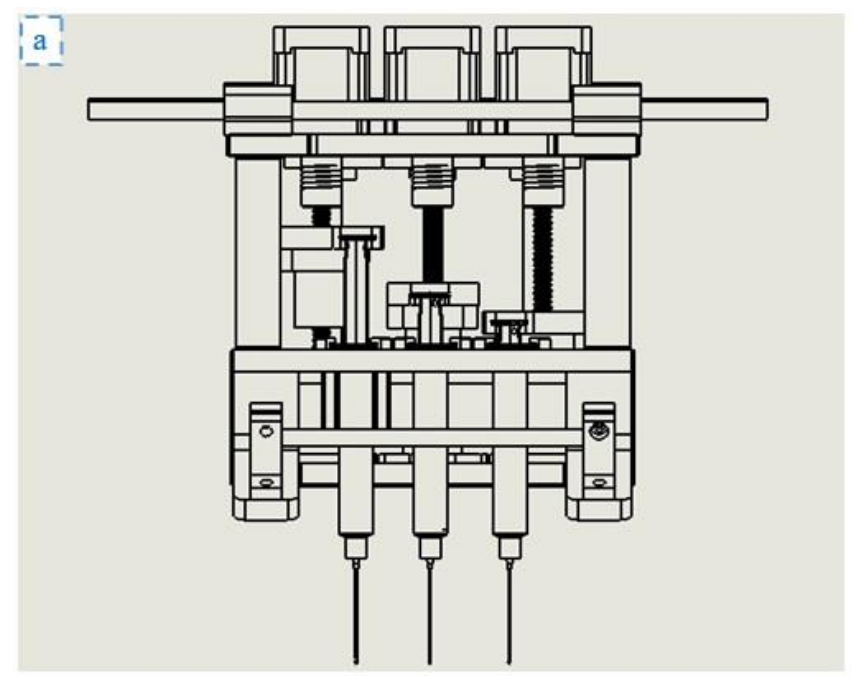




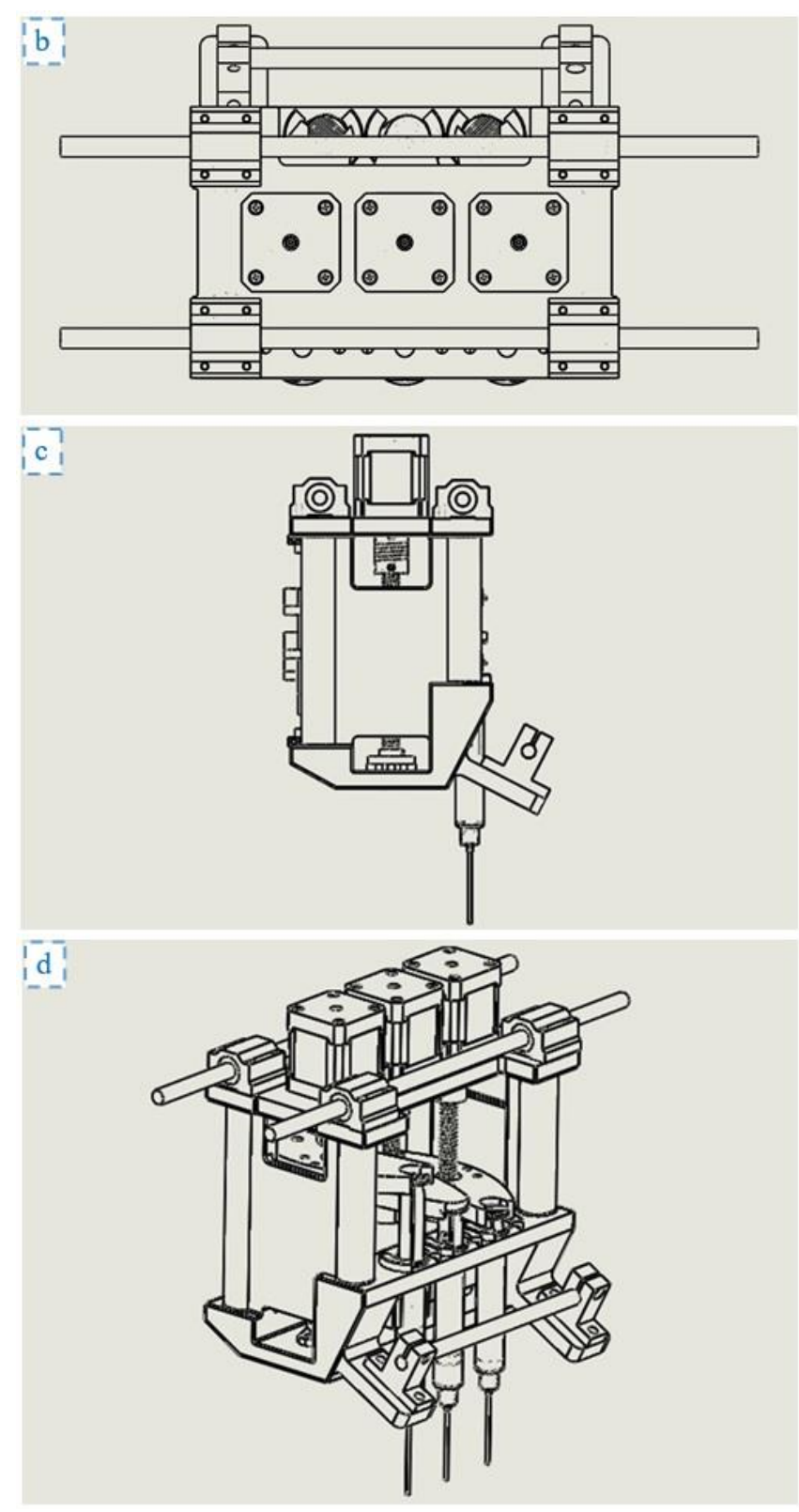

Figure 4. The compact micro-extrusion module: a) front view, b) top view, c) rear view, and d) perspective view [13]

Figure 5 illustrates the designed syringe holders.

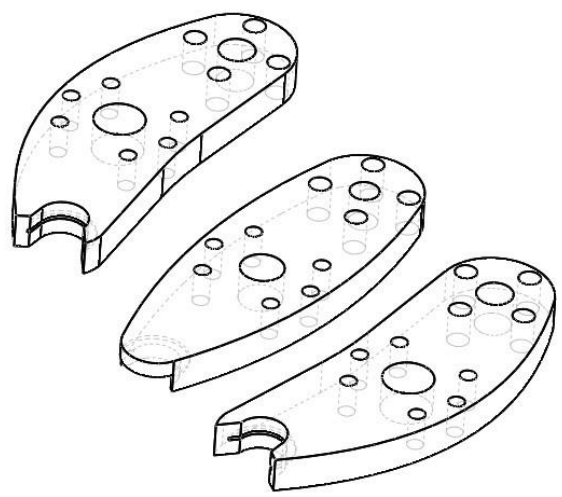

Figure 5. Syringe holders
Table 1 illustrates the BOM of the whole module.

Table 1. BOM of designed prototype

\begin{tabular}{|c|c|c|}
\hline Group & Piece(s) & Name \\
\hline \multirow{4}{*}{ Electrical } & 3 & Nema stepper motor \\
\cline { 2 - 3 } & 3 & Ceramic heater \\
\cline { 2 - 3 } & 3 & Thermocouple or thermistor \\
\hline \multirow{4}{*}{ Mechanical } & 4 & Steel rod \\
\cline { 2 - 3 } & 3 & Ball screw \\
\cline { 2 - 3 } & 3 & Ball screw nut \\
\cline { 2 - 3 } & 3 & Flexible coupling \\
\cline { 2 - 3 } & 4 & SCE UU series bearing \\
\cline { 2 - 3 } & 9 & SHF series bearing \\
\cline { 2 - 3 } & 3 & LMEF series bearing \\
\hline \multirow{5}{*}{$3 d$ printed } & 2 & SK series rod holder \\
\cline { 2 - 3 } & 3 & Main frame \\
\hline \multirow{3}{*}{ Other } & 3 & Syringe holder \\
\cline { 2 - 3 } & 3 & Syringe \\
\cline { 2 - 3 } & 3 & Steel blunt-ended needle \\
\hline
\end{tabular}

The main frame and syringe holders were manufactured from Polylactic acid (PLA) polymer with \% 60 infill rate and $200 \mu \mathrm{m}$ layer height by using a $3 d$ printer. Figure 6 illustrates the $3 d$ printed main frame.

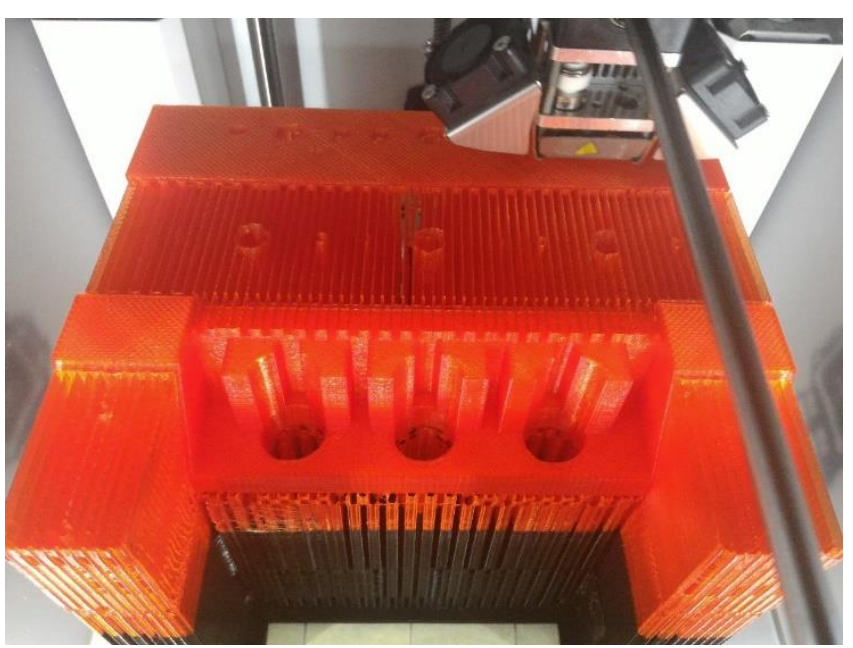

Figure 6. Main frame

Support parts are removed after the printing is completed. Figure 7 illustrates the assembly of the module. 


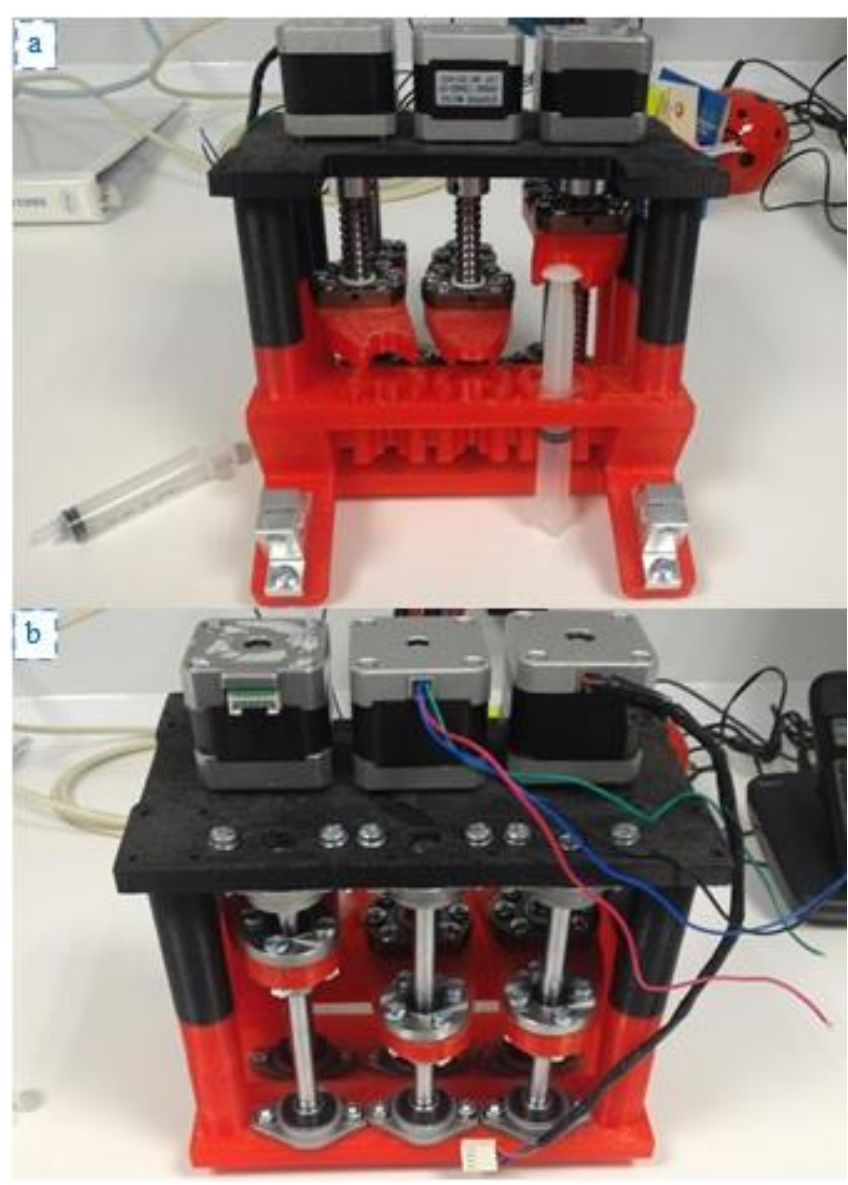

Figure 7. Assembly process of the module: front view a) and back view b).

\section{Conclusion}

The designed micro-extrusion module successively passed the bio-printing tests. Advantages of this system can be summarized as follows:

- Number of printing heads can be duplicated according to the needs and complexity of the target tissue constructs,

- Design can be scalable for different types of mechanical and electrical components,

- Steel rods support ball screw driven linear motions for precise micro-extrusion,

- Ergonomically, syringes can be easily mount and locked,

- System has laser holders that can be used for targeted photo polymerization,

- Heaters and temperature sensors can be easily mount,

- This module has compatibility for any type of a bioprinter.

First bio-printing tests were carried out on a bio-printer after integration of the designed module. In order to test durability and accuracy of this system, hybrid hydrogels of wide concentration range were prepared as bio-inks from natural polymers. Figure 8 illustrates the bio-printing process by means of the assembled micro-extrusion module.

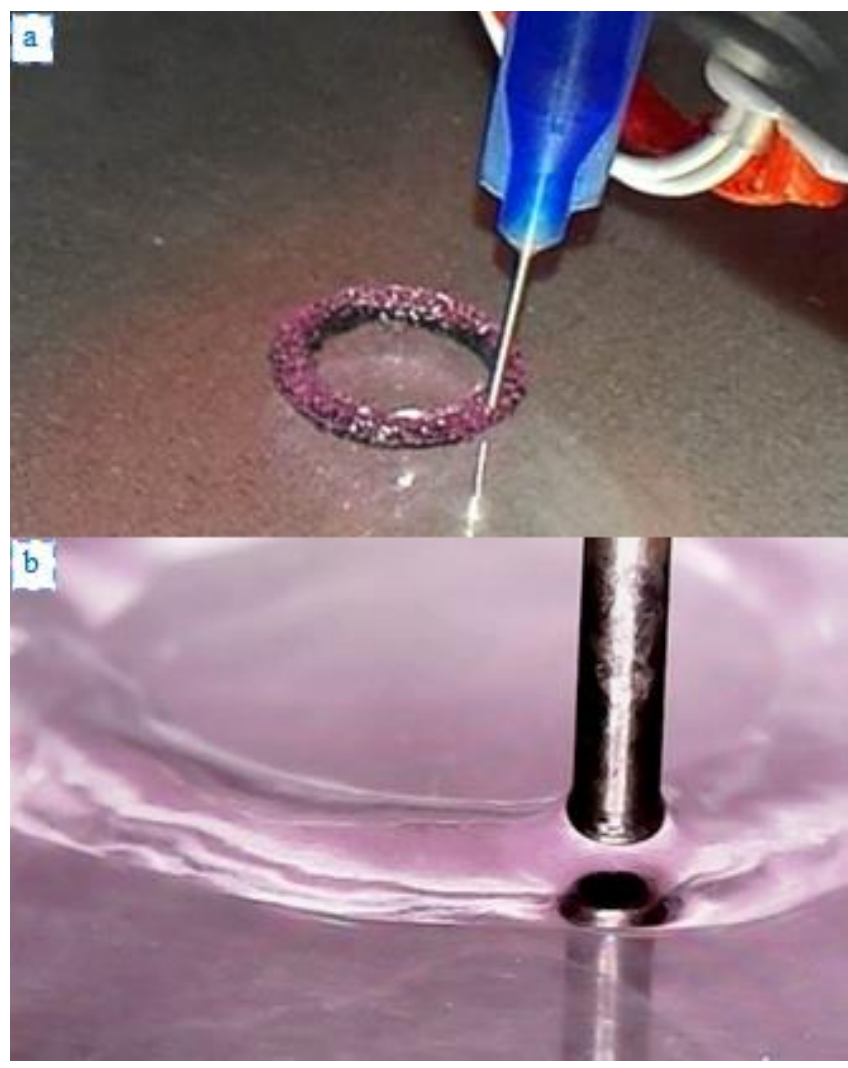

Figure 8. The bio-printing process by means of the assembled microextrusion module (24-gauge needle was used): a) without optimized bioprinting parameters and bio-ink b) with optimized bio-printing parameters and bio-ink. (hand-held microscope image)

\section{Acknowledgements}

This study was funded as a BAP Project by University of Kocaeli (Contract number: 2016/006).

\section{References}

[1] Murphy S. V. and Atala A. 3D bioprinting of tissues and organs, Nature biotechnology, Vol. 32, pp. 773-785.

[2] Hutchings I. M. and Martin G. D. Inkjet technology for digital fabrication. Chichester: Wiley, 2013.

[3] Aydın L., Küçük S., and Kenar H. Design and construction of a $3 \mathrm{~d}$ bioprinter for bioprinting of tissues and organs, Proceedings on the Tip Tekno 15, 2015, pp. 153-157.

[4] Chen H. and Özbolat İ. A multi-material bioprinting platform towards stratified articular cartilage tissue fabrication, Proceedings on the IIE Annual Conference, 2013, pp. 2246-2252.

[5] Panwar A. and Tan L. P. Current Status of Bioinks for Micro-Extrusion-Based 3D Bioprinting, Molecules, 2016, 21.6: 685 .

[6] Aydin L. Design and construction of ankle foot orthosis by means of three dimensional printers, M.S. thesis, Dept. Biomed. Eng., Kocaeli Univ., Kocaeli, Turkey, 2014.

[7] Akashi M., Akagi T. and Matsusaki M. Engineered Cell Manipulation for Biomedical Application, Springer Japan, 2014.

[8] Visscher D.O., et al. Advances in Bioprinting Technologies for Craniofacial Reconstruction, Trends in biotechnology, 2016.

[9] Obregon F., et al. Three-dimensional bioprinting for regenerative dentistry and craniofacial tissue engineering, 
Journal of Dental Research, 2015.

[10] Ozbolat I. T. and Hospodiuk M. Current advances and future perspectives in extrusion-based bioprinting, Biomaterials, Vol. 76, pp. 321-343.

[11] Blaeser A., et al. Controlling shear stress in 3D bioprinting is a key factor to balance printing resolution and stem cell integrity, Advanced healthcare materials, Vol. 5(3), pp. 326-333.

[12] Ferris C. J., Gilmore K. G. and Wallace G. G. Biofabrication: an overview of the approaches used for printing of living cells, Applied microbiology and biotechnology, Vol. 97(10), pp. 4243-4258.

[13] Aydin L. and Kenar H. Design and construction of a compact micro-extrusion module for bio-printing, TPE 2016/07571, June. 06, 2016. 